\title{
The DIAB-HYCAR study
}

\author{
Ph. Passa ${ }^{1}$, G . C hatellier ${ }^{2}$ on behalf of the D iab-H ycar Study G roup ${ }^{*}$ \\ ${ }^{1}$ D iabetes D epartment, Saint L ouis H ospital, Paris, France \\ 2 M edical I nformation D epartment, B roussais H ospital, Paris, France
}

Summary Microalbuminuria and proteinuria are strong independent predictors for increased cardiovascular mortality in non-insulin-dependent diabetic (NID D M ) patients. In such patients, angiotensin converting enzyme ( $A C E$ ) inhibition improves the evolution of diabetic nephropathy; however, no data are currently available on the effects of such intervention on cardiovascular morbidity and mortality. The aim of the $\mathrm{D}$ iab-H ycar study is to test the hypothesis that A CE inhibition with a low daily dose of $1.25 \mathrm{mg}$ ramipril, which has no significant effect on blood pressure, may reduce cardiovascular morbidity and/or mortality in normotensive or hypertensive N ID D M patients with persistent albuminuria. Selected and followed by general practitioners, 4000 patients will receive their usual oral antidiabetic treatment and if necessary antihypertensive treatment (A CE inhibitors excluded). In addition in a randomized, double-blind trial they will be given either a placebo or $1.25 \mathrm{mg}$ ramipril daily. The follow-up is currently scheduled to last 3 years. The efficacy of A CE-inhibition will be assessed by the following major end-points: cardiovascular death, sudden death, myocardial infarction, stroke, renal replacement therapy. The DiabH ycar study started on 3 February 1995. By 1 September 1995, 11000 urine samples were tested. The prevalence of persistent albuminuria was $23 \%, 964$ patients were initially included in the study, with 619 eligible patients included soon after. D ifferent strategies have been developed to record cardiovascular events correctly and to minimize the number of patients lost to follow-up. [D iabetologia (1996) 39: 1662-1667]

Keywords NIDDM, microalbuminuria, proteinuria, A CE -inhibition, cardiovascular morbidity and mortality.
Premature cardiovascular morbidity and mortality, mainly related to coronary heart disease ( $\mathrm{CHD}$ ) are major problems in non-insulin-dependent diabetic (NID D M ) patients. D ifferent large-scale, prospective epidemiological studies have shown that in NIDDM patients the incidence of deaths from cardiovascular

Corresponding author: Dr. Ph. Passa, D iabetes Department, Saint L ouis Hospital, 1 A venue Claude Vellefaux, F-75475 Paris, Cedex 10, France

* See A cknowledgements

A bbreviations: I N SE R M , Institut National de la Santé et de la Recherche M édicale; CNA M, Caisse Nationale d'A ssurance Maladie; $A C E$, angiotensin converting enzyme; $C H D$, coronary heart disease; NIDDM, non-insulin-dependent diabetes mellitus; C N I L , L a Commission I nformatique et L ibertès. disease in males is double the incidence in matched non-diabetic subjects and is even higher in females [1]. In the Multiple R isk Factor Intervention Trial, NIDDM appeared to be a strong independent risk factor for CHD in males [2]. In NIDD M patients, microalbuminuria is not only a marker for the risk of developing diabetic nephropathy, but is also a strong independent predictor of increased mortality, mainly attributable to cardiovascular diseases.

Since the publication by M ogensen in 1984 [3], numerous studies have confirmed that microalbuminuria is associated with an increased incidence of cardiovascular deaths $[4,5]$. M ac Leod et al. [6] have shown that this incidence also increased significantly in NIDDM patients with slightly elevated urinary albumin excretion, i.e. from 10.6 to $29.9 \mu \mathrm{g} / \mathrm{min}$, a 
range well beyond the common definition of microalbuminuria, compared to matched normoalbuminuric NIDDM patients.

Very few data are currently available on therapeutic interventions designed to reduce cardiovascular morbidity and mortality among NIDDM patients. No study has shown that improved glycaemic control has a long-term beneficial effect. In the double-blind, placebo-controlled study conducted by Ravid et al. [7] over a period of 5 years, angiotensin-converting enzyme (A CE) inhibition was observed to have a stabilizing effect on plasma creatinine and proteinuria in normotensive NIDDM patients. In a study by Lebovitz et al. [8] of 121 hypertensive NIDDM patients with various diabetic kidney diseases, 3 years of A CE inhibition was associated with a specific renal protective effect in addition to the lowering of blood pressure. N either of these studies included any data concerning cardiovascular mortality. To our knowledge, no specific trial has been reported to investigate the effects of antihypertensive treatment, with or without A CE inhibition, on the morbidity or mortality of NIDDM patients.

H owever, during the last few years, different studies $[9,10]$ have shown that in patients with cardiac insufficiency or myocardial infarction and reduced ejection fraction, ACE inhibition significantly reduced cardiovascular mortality, as well as the incidence of new coronary events. Finally, A CE inhibition may reduce left ventricular hypertrophy, which is associated with increased mortality in hypertensive patients [11].

$\mathrm{R}$ ationale for using $1.25 \mathrm{mg}$ ramipril daily. $\mathrm{R}$ amipril is an effective and safe A CE inhibitor, currently used worldwide at daily doses ranging from 1.25 to $10 \mathrm{mg}$. It has no undesirable metabolic side effects in NIDD M patients [12]. The A cute Infarction R amipil Efficiency Study [13], a double-blind placebo controlled study of patients with recent onset myocardial infarction complicated by cardiac insufficiency, has shown that the use of ramipril was associated with a significant reduction in mortality. A the low dose of $1.25 \mathrm{mg}$ daily, ramipril was shown to have no significant antihypertensive effects in animal models or humans [14].

In insulin-dependent diabetic patients with microalbuminuria, $1.25 \mathrm{mg}$ ramipril daily reduced urinary albumin excretion [15], and in hypertensive non-diabetic patients treated with furosemide, the addition of $1.25 \mathrm{mg}$ ramipril daily improved the regression of left ventricular hypertrophy [16]. Lastly, in a doubleblind placebo controlled, cross-over study conducted during a 4-week period, it was demonstrated in eight NIDDM patients that $24 \mathrm{~h}$ after ingestion of $1.25 \mathrm{mg}$ ramipril daily, this dose inhibited A CE activity as effectively as the dose of $5 \mathrm{mg}$ determined in vitro by Cushman's method, and in vivo by the angiotensin
II/angiotensin I ratio (unpublished data). In short, chronic administration of ramipril $1.25 \mathrm{mg}$ once a day inhibits A CE activity for $24 \mathrm{~h}$ after the last intake; has no significant antihypertensive effect; and has few side effects. The selection of this low dose is very important when investigating any specific effect of A CE inhibition on cardiovascular morbidity and mortality, separately from its effect of lowering blood pressure.

Consequently, the aim of the Diab-H ycar Study, now under way is to test the hypothesis that once daily A CE inhibition with a low dose of $1.25 \mathrm{mg}$ ramipril reduces morbidity and/or mortality in normotensive or hypertensive NIDDM patients with microalbuminuria or proteinuria.

\section{Feasibility study}

The final design of the protocol was preceded by a feasibility phase study [17]. During 1 month (1 N ovember - 1D ecember 1993) 792 general practitioners (GPs) were randomly selected and then contacted with the logistic support of $\mathrm{L}$ aboratoires $\mathrm{H}$ oechst France. GPs were asked to provide Le Centre Hospitalier $\mathrm{dA}$ ngers with random urine samples from NIDDM patients, collected twice within 6 weeks; 509 G Ps agreed to participate, and 326 worked actively and selected 2025 NIDDM patients whose urine was tested twice for albuminuria.

The prevalence of persistent microalbuminuria or proteinuria was $27.8 \%$ in this population. The mean age of NIDDM patients with microalbuminuria or proteinuria was $64.3 \pm 10.2$ years.

In summary, about $30 \%$ of the GPs contacted were active investigators and agreed to participate in a 3-year trial, and $80 \%$ of the patients with albuminuria agreed to be involved in such a trial. These data were encouraging and useful for the design of the final protocol.

II $\mathrm{H}$ ypotheses and calculation of sample size for the trial

There was no direct way of calculating the number of subjects to be included in the Diab-H ycar study since there is no French cohort study on the risk of death of NIDDM patients with microalbuminuria. The expected rate of death was therefore based on a review of the available literature about the risk of death in patients with microalbuminuria and published cohort studies. Prospective studies of clinic populations (reviewed in [5]) and of population-based-cohorts [4-6, 18] have showed that the relative risk of early mortality in patients with NIDDM and microalbuminuria was 2.2 to 4.0 compared to NIDDM patients without microalbuminuria. In various cohorts of middle-aged 
Diab-Hycar Study

- usual antidiabetic treatment

- if necessary antihypertensive treatment (ACE-I excluded)

2 determinations of urinary

albumin excretion (in Angers)

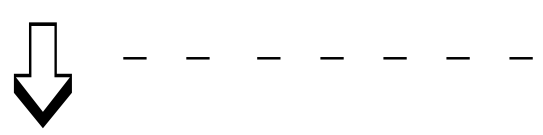

Months

Clinical examination

Biological determinations

Albuminuria

Cardiovascular morbidity, mortality

Fig. 1. see text

NID D M patients (mean age 52-66 years), most without microalbuminuria followed from 3 to 6.1 years, the cardiovascular mortality ranged from 2 to $13 \%$ [4-6, 18-21]. The low cardiovascular mortality rate in the $\mathrm{H}$ ypertension In Diabetes Study $[19,20]$ was explained by the inclusion of relatively young patients with recently discovered diabetes (less than 1 year). The high incidence in the ETDR S study [21] was due to the high proportion of patients with cardiovascular disease at inclusion. Taking into account these data and the characteristics of the expected population (most patients aged more than 60 years, frequently with hypertension and microalbuminuria), we anticipated a $12 \%$ total mortality rate corresponding to a 6-7\% cardiovascular mortality rate and to a $20 \%$ overall incidence of the principal endpoint (cardiovascular death, myocardial infarction, stroke and end-stage renal insufficiency).

The reduction in the number of these events in response to ramipril in NIDDM will probably reach a plateau due to the age of the subjects, as elderly patients may have other causes of cardiovascular or renal disease apart from diabetes. A s a relative reduction in the risk of morbid events would not have any clinical relevance below a threshold of $20 \%$, this threshold was adopted for the calculation of sample size.

A ssuming a $5 \% \alpha$ risk, 2000 subjects had to be included in each group to obtain a $90 \%$ power of detecting a $20 \%$ relative risk reduction. If the expected incidence of events is actually observed in the recruited population, 400 events will be observed in the placebo group, and 320 in the active treatment group.
Ramipril $1.25 \mathrm{mg} /$ daily $n=2000$

Placebo $n=2000$

$\begin{array}{cccccc}6 & 12 & 18 & 24 & 30 & 36 \\ \mathrm{x} & \mathrm{x} & \mathrm{x} & \mathrm{x} & \mathrm{x} & \mathrm{x} \\ & \mathrm{x} & & \mathrm{x} & & \mathrm{x} \\ & & & & & \mathrm{x} \\ \mathrm{x} & \mathrm{x} & \mathrm{x} & \mathrm{x} & \mathrm{x} & \mathrm{x}\end{array}$

III Protocol design (Fig. 1)

Selection of the G P s. From 10 ctober 1994 to 31 J anuary 1995, 4000 general practitioners from across France were selected for their special interest in diabetes, hypertension or clinical trials. To be selected, they had to agree to recruit two NIDDM patients with microalbuminuria or proteinuria within 1 year, and to follow them up for 3 years. These patients would receive their usual antidiabetic treatment, if necessary an antihypertensive treatment (excluding A CE inhibitors as well as angiotensin II receptor antagonists). In addition, in a randomized, double-blind trial, they will be given either a placebo or $1.25 \mathrm{mg}$ ramipril once a day. It was assumed that $50 \%$ of the 4000 G Ps would be active investigators, and would be able to include 4000 NIDDM patients with albuminuria or proteinuria in the trial.

\section{Patients}

Inclusion criteria: patients receiving oral antidiabetic agents (pragmatic definition of NIDD M)

- age 50 years or more, male or female

- with albuminuria $\geq 20 \mathrm{mg} / \mathrm{l}$, determined on two occasions by immunoturbidimetry at the Centre $\mathrm{Ho}$ spitalier d'A ngers in random urine samples

- serum creatinine $\leq 150 \mu \mathrm{mol} / \mathrm{l}$ (Jaffe's method)

- normotensive or hypertensive

- giving of written, informed consent and agreement to provide the investigator with their present address and the addresses of two close relatives. The recording of this information was approved by $L$ a Commission Informatique et Libertés (CNIL, France). 
Exclusion criteria: one of the following:

- treatment with insulin, an A CE inhibitor or an angiotensin II receptor antagonist

- urinary infection (Clinitek-dipstick test)

- cardiac insufficiency

- recent myocardial infarction, during the last

3 months

- previous intolerance to an A CE inhibitor

- chronic alcoholism or drug abuse, and inability to

understand the methods and goal of the trial

- poor life expectancy (cancer, A ID S, etc.)

- refusal to sign the informed consent to participate

in the trial.

Recording of clinical and biological parameters

A t inclusion, the following clinical information is recorded:

- name, body weight, height, body mass index

- known duration of diabetes

- blood pressure, using a mercury sphygmomanometer with the patient in the recumbent position

- any current treatments

- cardiovascular risk factors: smoking habits, hyperlipidaemia, duration of hypertension, familial premature cardiovascular diseases

- previous cardiovascular complications: myocardial infarction, angina, cerebrovascular complications, arteritis, laser photo-coagulation for diabetic retinopathy

- and finally, a resting 12-lead ECG.

A t inclusion, a $40 \mathrm{ml}$ blood sample is collected, sent to the Centre H ospitalier d'A ngers for D NA extraction, and stored with four aliquots of plasma and serum at $-80^{\circ} \mathrm{C}$ for determination of glycated haemoglobin, A CE activity, lipoproteins, etc.

A t inclusion in the trial, the G Ps provide the patients with the randomized treatment, i.e. placebo or ramipril, for a period of 6 months.

Consultations take place every 6 months and include a clinical examination similar to the initial one. A ny modification in the treatment regimen is recorded, as well as undesirable side effects, the incidence of new pathology and cardiovascular complications.

O nce a year, blood samples are collected for the determination of $\mathrm{H} \mathrm{bA}_{1 \mathrm{c}}$, serum creatinine and lipoproteins, and a random urine sample, for the determination of albuminuria. A resting $E$ CG is also performed.

The follow-up is currently scheduled to last 3 years. The efficacy of A CE inhibition will be assessed on the following endpoints.

M ajor endpoints:

- cardiovascular death, sudden death

- documented myocardial infarction
- stroke

- renal replacement therapy.

M inor endpoints:

- death, whatever the cause

- hospitalization for coronary angioplasty or bypass

revascularization

- documented cardiac insufficiency

- documented transient cerebrovascular ischaemia

- doubling of initial serum creatinine concentration

- loss of visual acuity in one eye

- peripheral amputation

Compliance with treatment

- compliance will be supervised by each investigator, who will record the cause and duration of treatment suspension; in cases of permanent cessation of treatment, data will be analysed for intention to treat.

This protocol was approved by the ethics committee of Centre H ospitalo-U niversitaire d'A ngers, and the study has been notified to the M edical A gency according to the current national regulations.

IV Study organization and functioning of the committees

This study is being carried out under the control of several committees whose functions are here described:

Scientific Steering Committee

- Finalization and validation of the protocol and case report forms for the principal study, and development and evaluation of ancilliary study protocols which must be specifically financed.

- Development of educational material designed for the training of investigators, followed by the publication of the study journal designed to maintain motivation throughout the study.

- In accordance with proposals of the Efficacy and Safety Surveillance Committee, the Scientific Steering Committee may prematurely suspend the trial, prolong the observation period or readjust the calculation of the sample size.

- Validation of analyses of the results of the study.

- A ppointment of editorial committees composed of members of the other study committees most able to provide relevant information and actively participate in the preparation of publications concerning the study (its methodology, state of progress, principal and secondary results, ancillary studies, etc). A ppointment of the authors of publications, giving preference to members of the editorial committees and participants in the ancillary studies, and relations with the medical press in general.

- Relations with the sponsor and health authorities (I NSE R M , CNA M , D rug A gency, French M inistry of 
H ealth, M inistry of R esearch) and scientific societies (E uropean and French D iabetology, Cardiology, $\mathrm{H}$ ypertension, Therapeutic and N ephrology societies).

Coordination and L ogistics Committee

- D esign and management of random treatment allocation by $M$ initel or vocal file server.

- Allocation of initial supplies to investigators, followed by the monitoring of subsequent drug supplies ensured by the Production U nit of Laboratoires Hoechst.

- D ata collection by recording, data entry, and verification of detachable pages of the case-report forms, which will allow real-time constitution of the file used to analyse the results of the study.

- Telephone and on-site monitoring of serious events and/or events constituting part of the morbidity-mortality criterion.

- A udit of at least $10 \%$ of the centres to ensure conformity with $\mathrm{G}$ ood Clinical Practice.

- Submission of all serious events and/or events constituting part of the morbidity-mortality criterion to the Clinical $E$ vent Validation Committee.

- Closure of centres.

- Conduct of the analyses defined in the study protocol.

Critical $\mathrm{E}$ vent Validation Committee

A ppointed and supervised by the Scientific Steering Committee, this Committee, composed of independent experts, must validate all serious events and/or events possibly constituting part of the morbiditymortality criterion.

Independent Patient L iaison C entre

The role of this centre is to investigate patients identified as being lost to follow-up by the investigators and by the Coordination and L ogistics Committee.

L ocation of the Centre. Centre R égional de Pharmacovigilance de Saint-E tienne, Professor M . O llagnier. C.H.R.U.Hôpital de B ellevue.

Clinical Pathology Committee. Responsible for the collection and storage of samples for laboratory tests, for conducting these tests, and for the sample bank of the study's laboratory.

E fficacy and Safety Surveillance Committee. Throughout the study, this committee will analyse the characteristics of the population of patients included, and the observed incidence of events corresponding to the assessment criteria for other events defined in the protocol. I t will give its opinion concerning the validity of the calculation of the sample size, and the probability of being able to obtain a valid result.

It will also interpret the analyses of the intermediate reports on efficacy, safety and serious events. It will provide the Scientific Steering Committee with recommendations concerning the continuation, prolongation or suspension of the study.

\section{P reliminary recruitment data}

The D iab-H ycar Study started on 3 February 1995. The study was announced at a Press Conference and a national meeting was held with 120 diabetologists, out of the 160 invited, who agreed to support a group of 20 to 30 local G Ps. O n account of the prevalence of microalbuminuria or proteinuria, in the population with NIDDM selected for the feasibility phase, it was estimated that urine samples from about 20000 NID D M patients would have to be tested twice in order to include 4000 NIDDM patients with persistent albuminuria in the study.

By 1 September 1995, 11000 urine samples had been received in the Centre Hospitalier d'A ngers. The prevalence of persistent albuminuria was $23 \%$, 964 N ID D M patients were included in the study, and 619 eligible NIDDM patients were included in the following weeks. This delay being due to summer vacations.

Of the G Ps 1900 proved to be active investigators. Most of them have only tested one or two patients for albuminuria. A few of them tested up to $20 \mathrm{pa}-$ tients for albuminuria and included more than two patients in the study.

\section{Discussion}

The above data, obtained in less than 7 months, are impressive because French doctors and patients are not accustomed to participating in large intervention trials lasting 3 years. A s it is currently estimated that the inclusion period will last for 20 to 24 months, the Scientific Steering Committee is developing new strategies to increase the rate of inclusion and to increase the number of investigators by 1500 . We remain confident that 4000 NID D M patients with albuminuria or proteinuria will eventually be included in the D iab-H ycar Study.

A nother problem is to stimulate patients and $G$ Ps to comply strictly with the protocol for 3 years. A bimonthly issue of the D iab-H ycar letter is sent to all participants in the study (patients and investigators) to improve their knowledge of NIDDM, hypertension, diabetic angiopathy, clinical trials, etc, and to provide them with the current inclusion data. Once a year, all the co-ordinators, i.e. the diabetologists, will meet their active investigators for discussion and stimulation. They will have to deal with problems, such as some G Ps being reluctant, for ethical reasons, to leave NIDDM patients who have albuminuria without A CE inhibition for 3 years despite the absence of scientific data indicating that this is harmful. 
In such a study, the correct recording of endpoints is crucial. A full-time doctor is visiting hospitals, clinics and families to obtain all the necessary medical information. The number of patients lost to follow-up must be as small as possible - 0 being the optimal goal. To obtain this goal, two full-time techniciansimmediately start trying to locate any patient who misses a consultation by telephone and letter. They may also contact the relatives who agreed to give their address prior to the patient's inclusion.

The Diab-H ycar Study provides an opportunity to perform a clinical prospective genetic study of a large number of NID D M patients at high risk of cardiovascular disease. It may be possible to correlate genotypes with patients' outcome and with the therapeutic effect of ramipril.

The Diab-H ycar Study may provide an answer to the crucial and still-debated question. Does longterm A CE inhibition reduce cardiovascular morbidity and mortality independently of its blood-pressure-lowering effect in NIDDM patients with albuminuria or proteinuria?

A cknowledgements. The D iab-H ycar Study is supported by a grant from and the technical assistance of the $L$ aboratoires $\mathrm{H}$ oechst (France), by I NSE R M , and L a C aisse Nationale d'A ssurance $M$ aladie. The French Societies for the study of nephrology, hypertension, and diabetes have given it their approval and will be informed annually about the progress. Professor $M$. $M$ arre (A ngers) is the principal investigator.

Composition of the different committees involved in the D iab$\mathrm{H}$ ycar Study.

Scientific Steering Committee

F. A lhenc-G elas, F. Cambien, J.P. B oissel, A . G irault-L ouvel, P. G ueret, M. M arre (Principal Investigator), J. M enard (V ice Chairman), Ph. Passa (Chairman), P.F. Plouin, D. Vasmant (Secretary), Cl. Weisselberg.

Coordination and L ogistics Committee

J.P. B oissel (Chairman), M. Marre, M. Lievre (Vice-Chairman), P. F. Plouin, J. C. R eglier (Secretary), D. Vasmant, F. G uenaneche.

Critical E vent Validation Committee

G. Chatellier (Chairman), Th. G abreau, Ph. Fressinaud, H . L eblanc, M. A zizi.

Clinical Pathology Committee

F. A lhenc-G elas (Chairman), F. Cambien, A. G irault-L ouvel (Vice-Chairman), M. Marre, J. M enard, M. L ievre.

E fficacy and Safety Surveillance Committee

E. E schwege, S. Weber, N. Victor, C. E. M ogensen.

\section{References}

1. K annel WB, M CG ee DL (1979) D iabetes and cardiovascular risk factors. The Framingham Study. Circulation 59: 8-13

2. Stamler J, Vaccaro O, Neaton J D, Wentworth D (1993) For the Multiple Risk Factor Intervention Trial Research Group $D$ iabetes, other risk factors and 12-year cardiovascular mortality for men screened in the M ultiple R isk Factor Intervention Trial. D iabetes Care 16: 434-444

3. Mogensen CE (1984) Microalbuminuria predicts clinical proteinuria and early mortality in maturity onset diabetes. N Engl J Med 310: 356-360
4. M attock $M B$, M orrish $\mathrm{N}$ J, Viberti $\mathrm{GC}$, K een $\mathrm{H}$, Fitzgerald A P, Jackson G (1992) Prospective study of microalbuminuria as predictor of mortality in NIDDM. Diabetes 41 : 736- 741

5. N eil A, Hawkins M, Potok M, Thorogood M, Cohen D, Mann J (1993) A prospective population-based study of microalbuminuria as a predictor of mortality in NIDDM . Diabetes Care 16: 996-1003

6. M acL eod J M, L utale J, M arshall SM (1995) A lbumin excretion and vascular deaths in NIDDM. Diabetologia 38: 610-616

7. $R$ avid $M$, Savin $H$, J utrin $I, B$ ental $T, K$ atz $B$, L ishner $M$ (1993) L ong-term stabilizing effect of angiotensin converting enzyme inhibition on plasma creatinine and on proteinuria in normotensive type II diabetic patients. A nn Int M ed 118: 577-581

8. Lebovitz HE, W iegmann TB, Cnaan A et al. (1994) Renal protective effects of enalapril in hypertensive NIDDM. R ole of baseline albuminuria. Kidney Int 45:[Suppl] S150S155

9. Pfeffer MA (1992) Effect of captopril on mortality and morbidity in patients with left ventricular dysfunction after myocardial infarction: results of the survival and ventricular enlargement trial. N E ngl J M ed 322: 669-677

10. Y usuf S (1992) E ffect of enalapril on myocardial infarction and unstable angina in patients with low ejection fraction. Lancet 340: 1173-1178

11. D ahlof B, Pennert K, H ansson L (1992) R egression of left ventricular hypertrophy. A meta-analysis. A $\mathrm{m} \mathrm{J} \mathrm{H}$ ypertens 5: $95-110$

12. Janka HU, N uber A, M enhert H (1990) M etabolic effect of the treatment by ramipril in hypertensive patients with NID D M . D rug R es 40: 432-435

13. The acute infarction ramipril efficacy (AIRE) study investigators (1993) E ffects of ramipril on mortality and morbidity of survivors of acute myocardial infarction with clinical evidence of heart failure. L ancet 342: 821-828

14. Vasmant $D, B$ ender $N$ (1989) The renin-angiotensin system and ramipril, a new converting enzyme inhibitor. J Cardiovas Pharmacol 14: 48-61

15. M arre M, Hallab M, Billard A et al. (1991) Small doses of ramipril to reduce microalbuminuria in diabetic patients with incipient nephropathy independently of blood pressure changes.] Cardiovasc Pharmacol 18[Suppl 2]: S165-S168

16. Lievre M, G ueret P, D elair S, B oissel JP (1995) O n behalf of the $\mathrm{H}$ ycar Study G roup. H ypertens 25: 92-97

17. M arre M , G irault A , Vasmant D au nom du Comité Scientifique du G roupe D iab-H ycar (1995) Prévalence de la microalbuminurie chez les diabétiques de type 2 français suivis par leur médecin généraliste. $D$ iabete $M$ etab 21: 34-40

18. Stiegler H, Standl E, Schulz K, R oth R, Lehmacher W (1992) Morbidity, mortality, and albuminuria in type 2 diabetic patients: a three-year prospective study of a random cohort in general practice. Diabet $M$ ed 9: 646-653

19. The $\mathrm{H}$ ypertension Study $\mathrm{G}$ roup (1993) $\mathrm{H}$ ypertension in D $\mathrm{i}$ abetes Study: I. Prevalence of hypertension in newly presenting type 2 diabetic patients and the association with risk factors for cardiovascular and diabetic complications. J H ypertens 11: 309-317

20. The $\mathrm{H}$ ypertension in D iabetes Study G roup (1993) H ypertension In Diabetes Study: II . Increased risk of cardiovascular complications in hpertensive type 2 diabetic patients. J Hypertens 11: 319-325

21. ETDR S Investigators (1992) A spirin effects on mortality and morbidity in patients with diabetes mellitus. Early treatment Diabetic R etinopathy Study Report 14. JA M A 268: $1292-1300$ 